Ann. Biol. anim. Bioch. Biophys., I968, 8 (4), 557-563.

\title{
DOSAGE DES ACIDES AMINES ET AMINES PAR LA NINHYDRINE. AMÉLIORATION PRATIQUE
}

\author{
M. C. MICHEL( $(\mathbf{)}$ \\ avec la collaboration technique de Geneviève HaNNEQUART \\ Station centrale de Nutrition, \\ Centre national de Recherches zootechniques, 78 - Jouy-en-Josas \\ Institut national de la Recherche agronomique
}

\section{SOMMAIRE}

Les réactifs employés pour le dosage colorimétrique des acides aminés comprennent :

a) une solution de ninhydrine $(20 \mathrm{~g})$ dans le méthylcellosolve $(500 \mathrm{ml})$, et un tampon propionate de sodium (201,8 g) et acide propionique $(93 \mathrm{ml}) ; \mathrm{H}_{2} \mathrm{O}$ q.s. I litre ;

b) une solution d'acide ascorbique à $\mathrm{I}$ mg par $\mathrm{ml}$ dans l'eau.

La solution à doser $(\mathrm{r} \mathrm{ml})$ est additionnée de $\mathrm{I} \mathrm{ml}$ de réactif $(a)$ et de 2 gouttes de réactif $(b)$. Après I 5 minutes au bain-marie bouillant, on ajoute $15 \mathrm{ml}$ d'alcool à $5 \circ \mathrm{p}$. roo et on lit la coloration à $570 \mathrm{~m} \mu$.

Les résultats obtenus indiquent :

a) une grande stabilité du réactif $(a)$, qui se conserve plus de 3 mois en présence d'air;

b) une excellente reproductibilité de la coloration;

c) un pouvoir tampon élevé du réactif, qui permet d'analyser des effluents de $\mathrm{pH}$ variés sans correction;

d) une réaction colorée avec les amines primaires très proche de celle des acides aminés correspondants.

\section{INTRODUCTION}

La formation d'un dérivé coloré entre la ninhydrine (hydrate de tricétohydrindène), et les acides aminés est connue depuis longtemps. L'intensité de la coloration, la stabilité d'un complexe coloré, sont déterminées par les conditions physicochimiques du milieu réactionnel, et en particulier par la présence de ninhydrine réduite (MOORE et STEIN, I948).

(1) Station de Physiopathologie de la nutrition Centre de Recherches zootechniques et vétérinaires sur les Ruminants 63 -Theix de Clermont-Ferrand.

Annales de Biologie animale. - rg68. 
Les réactifs proposés peuvent être classés en trois groupes :

a) La ninhydrine réduite (hydrindantine), est obtenue dans le réactif, à l'aide d'un réducteur de la ninhydrine. Celui-ci peut être le chlorure stanneux (MOORE et Stein, I948; Spackman et al., I958 ; Piez et Morris, I960) ; le cyanure de potassium (Troll et Cannan, I953; Yemm et Coking, I955; Kalant, I956); le cyanure de sodium (CADAvid et PALAdINI, I964 Rosen, I957) ; 1'acide ascorbique (YamaGASHI, I953) ; la dithionite et le fluorure de sodium (STEGEMAN, I960).

b) On ajoute au réactif une certaine proportion d'hydrindantine, synthétisée par réduction de la ninhydrine par l'acide ascorbique (MOORE et STEIN, I954; MaTHEson, rg6r; CONNEL et al., I955).

c) Le réactif à la ninhydrine - sans réducteur - est mélangé au liquide à doser contenant le réducteur (ROSEN et al., I962).

Les deux premières techniques conduisent à des réactifs instables, devant être conservés en l'absence totale d'oxygène. On observe souvent une formation de cristaux qui grippent les seringues des pipettes automatiques. Le blanc-réactif est variable et souvent élevé, surtout en présence de tampon phosphate.

Le réactif de ROSEN et al. (Ig62) ne présente pas ces inconvénients. Il est parfaitement stable et peut être conservé en présence d'air. Cependant, le réducteur proposé (KCN) n'étant pas suffisamment stable dans nos conditions expérimentales, nous avons mis au point un réactif modifié, destiné à l'analyse des amines biologiques.

\section{I. - RÉACTIFS}

\section{Réactif à la ninhydrine}

Dans un bécher de 2 litres placé sur un agitateur magnétique, on ajoute successivement :
a) acide propionique (Merck) .............. $93 \mathrm{ml}$
b) méthylcellosolve (Touzart) $\ldots \ldots \ldots \ldots \ldots \ldots, \quad 500 \mathrm{ml}$
c) propionate de sodium (Merck) .......... $201,8 \mathrm{~g}$
d) ninhydrine (Touzart) $\ldots \ldots \ldots \ldots \ldots \ldots \ldots, \quad 20 \mathrm{~g}$

Le cellosolve, stocké à $+4^{\circ} \mathrm{C}$ en flacons jaunes, est distillé avant l'emploi sous pression réduite.

\section{Solution d'acide ascorbique (Prolabo)}

A I $\mathrm{mg} / \mathrm{ml}$ dans l'eau.

\section{Acides aminés et amines (Calbiochem) (Eastmann)}

Solutions M/ Ioo dans l'eau. Elles sont ajustées à $\mathrm{pH} 6$ si nécessaire. Conserver à $-20^{\circ} \mathrm{C}$. Diluer au moment de l'emploi.

$$
\text { 4. L'eau }
$$

Employée pour toutes les solutions, l'eau est d'abord permutée, puis distillée dans un appareil métallique, et enfin désionisée sur une colonne de résine (SAGEI A 2o). Sa teneur en ammoniac est pratiquement nulle, ce qui permet d'obtenir un blanc réactif très faible. 


\section{II. - TECHNIQUE}

Dans des tubes Pyrex de $\mathrm{I} 6 \times \mathrm{I} 60 \mathrm{~mm}$, contenant la solution à doser $(\mathrm{r} \mathrm{ml})$, ajouter $\mathrm{I}$ goutte de la solution d'acide ascorbique à l'aide d'une pipette calibrée à 20 gouttes par ml, puis avec la seringue, I $\mathrm{ml}$ de réactif à la ninhydrine. Les placer ${ }_{5}$ minutes dans un bain-marie bouillant, puis les refroidir sous le robinet. Ajouter ensuite $15 \mathrm{ml}$ d'un mélange éthanol-eau (V/V). Agiter les tubes pour détruire l'excès d'hydrindantine. Le photomètre utilisé pour la lecture était un Unicam SP 1300 muni d'un filtre Ilford n $\mathrm{n}^{\circ} 624(495-575 \mathrm{~m} \mu)$, et d'une cuve de $\mathrm{I} \mathrm{cm}$. Toutes les lectures sont effectuées par rapport à l'eau, ce qui permet de contrôler la qualité des réactifs. Les valeurs indiquées sont rapportées à la coloration fournie par une micromole de la substance considérée.

\section{III. - EXPÉRIENCES PRÉLIMINAIRES}

a) La méthode de ROSEN et al. (I962), appliquée à l'analyse des amines après séparation sur colonne de résine, ne nous a pas donné de résultats satisfaisants. Dans les fractions ayant séjourné une nuit à 1'air, on observait une baisse du blanc-réactif, lequel était élevé au départ, et une réaction colorée non quantitative avec les amines et l'ammoniac.

Ceci nous a amené à utiliser un autre réducteur, 1'acide ascorbique, et à ajouter ce dernier au moment du dosage.

TABLEAU I

Relation entre la quantilé du réducteur et la formation du complexe coloré

\begin{tabular}{c|c|c}
\hline \multirow{2}{*}{$\begin{array}{c}\text { Acide ascorbique } \\
\mathrm{mg} / \text { tube }\end{array}$} & \multicolumn{2}{|c}{ Densité optique $\times 10^{3}$} \\
\cline { 2 - 3 } & Blanc & $\mathrm{N}\left(\mathrm{NH}_{8}\right) 1 \mu \mathrm{M}$ \\
\hline & & \\
\hline 0 & 15 & $56^{*}$ \\
10 & 16 & 640 \\
20 & 16 & 830 \\
30 & 17 & 975 \\
40 & 17 & 1000 \\
50 & 18 & 1001 \\
60 & 18 & 1001 \\
& & \\
\hline
\end{tabular}

(1) La valeur du blanc est déduite.

La quantité d'acide ascorbique nécessaire pour obtenir le maximum de coloration est de l'ordre de $50 \mu \mathrm{g}$ par $\mathrm{ml}$. Le blanc-réactif est très faible et reste pratiquement indépendant de la quantité de réducteur (tabl. I). L'essai reporté a été effectué avec l'ammoniac, la coloration fournie par ce dernier étant très sensible à la quantité d'hydrindantine présente dans le milieu réactionnel. 
Cette quantité de réducteur $(50 \mu \mathrm{g} / \mathrm{ml})$ est suffisante pour une teneur en azote aminé de l'ordre de 3 micromoles/ml et doit être augmentée pour des teneurs plus élevées (fig. I). Les valeurs indiquées sont obtenues par dilution de la solution à doser avant la lecture.

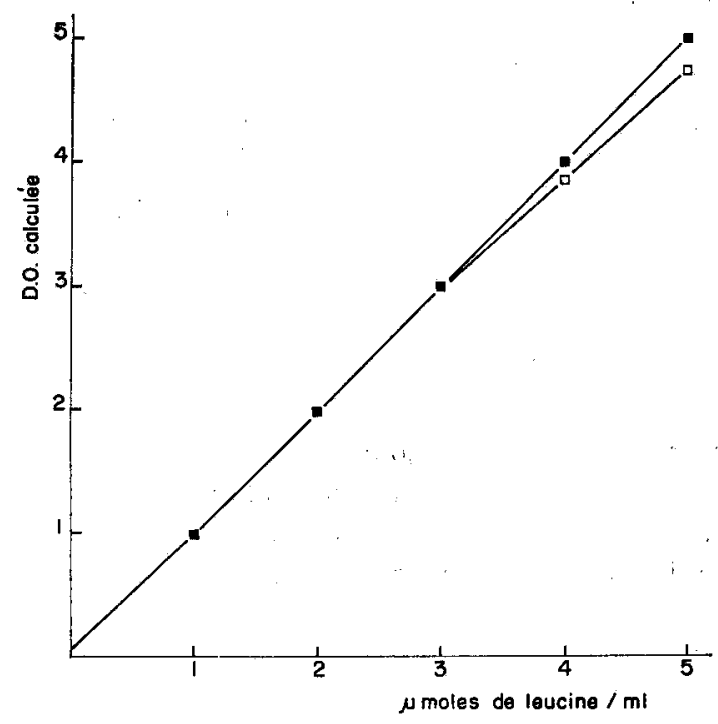

FIG. I. - Infuence du réducteur

ㅁ $50 \mu \mathrm{g} \mathrm{A.} \mathrm{Ascorbique/ml.} \mathrm{.} \mathrm{I} 100 \mu \mathrm{g} \mathrm{A.} \mathrm{Ascorbique} / \mathrm{ml}$.

TABLEAU 2

Infuence du $p H$ sur le développement de la coloration

\begin{tabular}{l}
\hline \\
\\
\end{tabular}

(

( ${ }^{2}$ La valeur du blanc réactif a été déduite.

b) Le pH du milieu réactionnel joue un rôle important sur le développement de la coloration. Dans la réaction étudiée par MOORE et STEIN (1948), le maximum de coloration n'était pas obtenu au même $\mathrm{pH}$ pour la leucine, le tryptophane et l'ammo- 
niac. Or, dans l'analyse chromatographique des acides aminés et amines, les effluents de colonne petuvent présenter une gamme variée de $\mathrm{pH}$ et de concentration saline. $\mathrm{I}$ est préférable dans la pratique de disposer d'un pouvoir tampon suffisant, plutôt que d'ajuster le $\mathrm{pH}$ du liquide à doser.

L'expérience montre que le réactif est très tamponné, et il n'est nécessaire de corriger le $\mathrm{pH}$ que pour des solutions très acides (tabl. 2). Les tampons usuels fournissant des valeurs pratiquement identiques.

\section{IV. - RÉSULTATS}

a) La détermination du coefficient de coloration propre à chaque substance est effectuée sur des solutions contenant $0-0, \mathrm{I}-0,2-0,4$ et 0,5 micromoles de celle-ci,

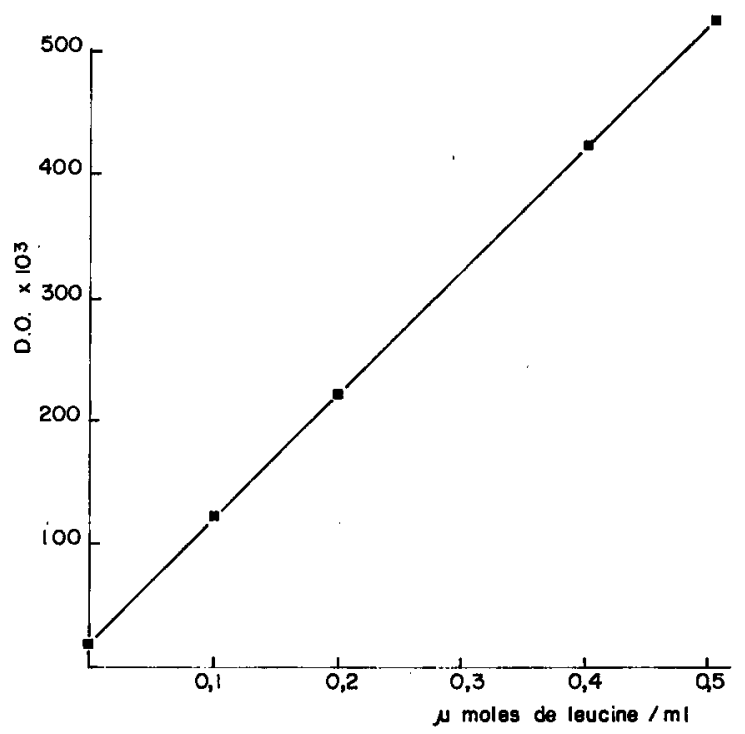

FIG. 2. - Dosage de la leucine

TABLEAU 3

Analyse d'un étalon leucine

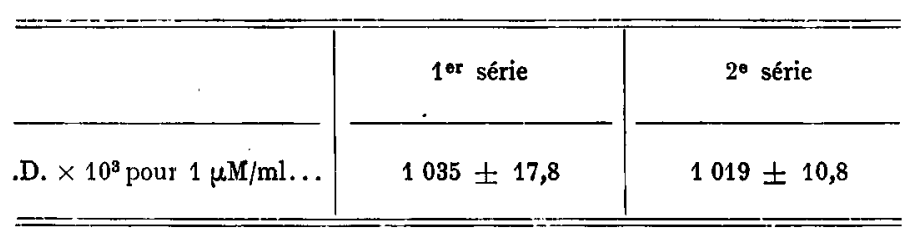

afin de rester dans les limites dans lesquelles la lecture photométrique fournit une droite en fonction de la concentration (fig. 2). 
b) La reproductibilité de la méthode est satisfaisante, d'après les résultats obtenus sur deux séries de 9 analyses effectuées à 6 mois d'intervalle (tabl. 3).

\section{TABLEAU 4}

Facteurs de coloration obtenus avec des acides aminés, amines, et substances diverses, rapportés à la leucine $=100$

\begin{tabular}{|c|c|c|c|}
\hline Leucine... . . . . . . . . . & 100 & $\mathrm{NH}_{\mathrm{a}}$ & 97 \\
\hline Méthionine $\ldots \ldots \ldots \ldots \ldots \ldots \ldots$ & 99 & 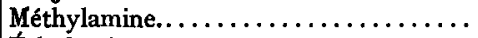 & 98 \\
\hline Glycine $\ldots \ldots \ldots \ldots \ldots \ldots \ldots \ldots$ & 94 & Éthylamine $\ldots \ldots \ldots \ldots \ldots \ldots$ & 98 \\
\hline Alanine..$\ldots \ldots \ldots \ldots \ldots \ldots \ldots$ & 103 & Propylamine $\ldots \ldots \ldots \ldots \ldots \ldots$ & 98 \\
\hline Isoleucine $\ldots \ldots \ldots \ldots \ldots \ldots \ldots \ldots$ & 101 & Butylamine $\ldots \ldots \ldots \ldots \ldots \ldots \ldots$ & 100 \\
\hline Phénylalanine.............. & 97 & Ethanolamine $\ldots \ldots \ldots \ldots \ldots \ldots$ & 92 \\
\hline Valine $\ldots \ldots \ldots \ldots \ldots \ldots \ldots \ldots$ & 100 & Leucamine $\ldots \ldots \ldots \ldots \ldots \ldots \ldots$ & 100 \\
\hline 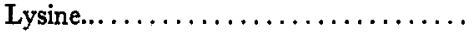 & 109 & Putrescine ..... & 115 \\
\hline 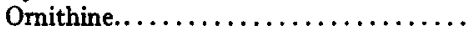 & 112 & Phénylalamine.......... & 97 \\
\hline Arginine $\ldots \ldots \ldots \ldots \ldots \ldots \ldots \ldots$ & 97 & Tyramine $\ldots \ldots \ldots \ldots \ldots \ldots \ldots$ & 97 \\
\hline Histidine $\ldots \ldots \ldots \ldots \ldots \ldots \ldots \ldots$ & 95 & Allylamine..$\ldots \ldots \ldots \ldots \ldots \ldots \ldots$ & 87 \\
\hline Tryptophane................. & 90 & Histamine $\ldots \ldots \ldots \ldots \ldots \ldots \ldots$ & 77 \\
\hline Acide aspartique $\ldots \ldots \ldots \ldots \ldots \ldots$ & 94 & Agmatine...$\ldots \ldots \ldots \ldots \ldots \ldots$ & 91 \\
\hline Acide glutamique $\ldots \ldots \ldots \ldots \ldots \ldots$ & 99 & 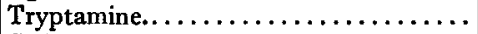 & 72 \\
\hline 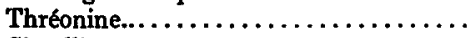 & 95 & Cadavérine $\ldots \ldots \ldots \ldots \ldots \ldots \ldots$ & 108 \\
\hline Citrulline $\ldots \ldots \ldots \ldots \ldots \ldots \ldots \ldots$ & 111 & Ansérine $\ldots \ldots \ldots \ldots \ldots \ldots \ldots \ldots$ & 84 \\
\hline Sérine....................... & 96 & Carnosine $\ldots \ldots \ldots \ldots \ldots \ldots \ldots \ldots$ & 84 \\
\hline Tyrosine $\ldots \ldots \ldots \ldots \ldots \ldots \ldots$ & 98 & Acide $\delta$-amino-valérique.......... & 94 \\
\hline 1-méthylhistidine $\ldots \ldots \ldots \ldots \ldots \ldots$ & 92 & Acide $\gamma$-amino-butyrique ........... & 95 \\
\hline 3-méthylhistidine ............... & 94 & Urée $\ldots \ldots \ldots \ldots \ldots \ldots$ & 0,03 \\
\hline Asparagine $\ldots \ldots \ldots \ldots \ldots \ldots \ldots$ & 94 & Guanidine.................. & 0 \\
\hline Glutamine $\ldots \ldots \ldots \ldots \ldots \ldots \ldots \ldots$ & 99 & Acide uréidosuccinique............. & $\mathbf{0}$ \\
\hline 5-hydroxytryptophane $\ldots \ldots \ldots \ldots \ldots$ & 81 & Diéthylamine + (jaune-vert) $\ldots \ldots \ldots$ & \\
\hline Glucosamine $\ldots \ldots \ldots \ldots \ldots \ldots \ldots$ & 93 & & \\
\hline
\end{tabular}

c) Les facteurs de coloration sont indiqués dans le tableau 4.

\section{CONCLUSION}

Le but de cette mise au point était d'obtenir une réaction colorée aussi sensible que possible avec les amines biologiques et l'ammoniac, pour leur analyse par chromatographie sur colonne de résine. Les résultats montrent que ces substances fournissent une coloration, sur une base équimoléculaire, très voisine des acides aminés correspondants.

Le pouvoir tampon élevé du réactif, qui permet d'analyser des effluents de $\mathrm{pH}$ varié sans correction, la faible valeur du blanc-réactif, qui n'est influencé ni par le $\mathrm{pH}$, ni par la concentration saline de la solution à doser, sont également des facteurs favorables.

Enfin, la conservation du réactif effectué sans précautions spéciales pendant plus de trois mois, permet d'envisager des analyses discontinues avec une bonne reproductibilité. 


\section{SUMMARY}

\section{QUANTITATIVE ESTIMATION OF AMINO-ACIDS AND AMINES BY NINHYDRINE} EXPERIMENTAL IMPROVEMENTS

The reagents used for colorimetric estimation of amino-acids are :

(a) $20 \mathrm{~g}$ ninhydrine dissolved in $500 \mathrm{ml}$ methyl Cellosolve, with a sodium propionate buffer (20r.8 g) and $93 \mathrm{ml}$ propionic acid, brought to I liter with $\mathrm{H}_{2} \mathrm{O}$.

(b) I $\mathrm{mg}$ ascorbic acid dissolved per $\mathrm{ml}$ water.

The solution to estimate $(\mathrm{I} \mathrm{ml})$ is added with $\mathrm{I} \mathrm{ml}$ reagent $(a)$ plus $0.1 \mathrm{ml}$ reagent $(b)$. After $15 \mathrm{mn}$ in a boiling water bath, $15 \mathrm{ml}$ of $5^{\circ}$ per cent aqueous ethanols is added and coloration recorded at $570 \mathrm{~m} \mu$.

Our results show.

I. A good stability of reagent (a), which can be kept for more than three months in contact with the air.

2. An excellent reproducibility of coloration.

3. A high buffer activity that allows the analysis of effluents of various $\mathrm{pH}$ values without corrections.

4. Coloration with primary amines very similar to that of the corresponding amino-acids.

\section{RÉFÉRENCES BIBLIOGRAPHIQUES}

Cadavid N. G., Paladini A. C., 1964. Automatic amino-acid analysis : reagent and instrumental improvements. Analyt. Biochem., 8, I70-1 74 .

Connell G. E., Dixon G. H., Hanes C. S., I955. Quantitative chromatographic methods for the study of enzymic transpeptidation reactions. Can. J. Biochem. Physiol., 38, 416-427.

KaLANT H., 1956. Colorimetric ninhydrin reaction for measurement of alpha amino nitrogen. Anal. Chem., 28, $265-266$.

Matheson A. T., Tigrane E., Hanes C. S., r96r. Quantitative chromatographic methods. 5. An improved ninhydrin hydrindantin reagent. Can. J. Biochem. Physiol., 39, 41 7-425.

MOORE S., SteIN W. H., I948. Photometric ninhydrin method for use in the chromatography of aminoacids. J. Biol. Chem., 176, 367-388.

MoORE S., STEIN W. H., I954. A modified ninhydrin reagent for the photometric determination of amino acids and related compounds. J. Biol. Chem., 211, 907-913.

PIEz K. A., Morris I., 1960. A modified procedure for the automatic analysis of amino acids. Anal. Biochem., 1, 187-201.

Rosen H., 1957. A modified ninhydrin colorimetric analysis for amino acids. Arch. Biochem. Biophys., 67, $10-15$.

Rosen H., Berard C. W., Levenson S. M., I962. A simplified procedure for automatic amino acid analysis. Analyt. Biochem., 4, 213-221.

SpackmanN D. H., STejN W. H., Moore S., 1958. Automatic recording apparatus for use in chromatography of amino acids. Anal. Chem., 80, I I90-1206.

StEgEmanN H., 1960. Bestimmung von Aminosäuren mit dithionitradieziertem Ninhydrin. HoppeSeyler's Z. Physiol. Chem., 318, 102-109.

Troll. W., Cannan R. K., I953. A modified photometric ninhydrin method for the analysis of amino and imino acids. J. Biol. Chem., 200, 803-81 1.

Yamagishi M., Yoshida T., T., I953. Studies on ninhydrin reaction. I. Colorimetric determination of $\alpha$-amino acids. J. Pharm. Soc. Japan, $73,675-676$.

Yemm E. W., Cocking E. C., I955. The determination of amino acids with ninhydrin. Analyst., 80, 209-2 I3. 